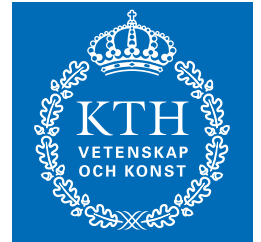 \\ KTH Electrical Engineering \\ Channel Quantization Design in Multiuser MIMO Systems: Asymptotic versus Practical Conclusions
}

Proceedings of IEEE International Conference on Acoustics, Speech, and Signal Processing (ICASSP).

22-27 May, Prague, Czech Republic, 2011

Copyright 2011 IEEE. Published in the IEEE 2011 International Conference on Acoustics, Speech, and Signal Processing (ICASSP 2011), scheduled for 22-27 May 2011 in Prague, Czech Republic. Personal use of this material is permitted. However, permission to reprint/republish this material for advertising or promotional purposes or for creating new collective works for resale or redistribution to servers or lists, or to reuse any copyrighted component of this work in other works, must be obtained from the IEEE. Contact: Manager, Copyrights and Permissions / IEEE Service Center / 445 Hoes Lane / P.O. Box 1331 / Piscataway, NJ 08855-1331, USA. Telephone: + Intl. 908-562-3966.

\section{EMIL BJÖRNSON, KONSTANTINOS NTONTIN, AND BJÖRN OTTERSTEN}

\author{
Stockholm 2011
}

\author{
KTH Royal Institute of Technology \\ ACCESS Linnaeus Center \\ Signal Processing Lab
}

DOI: 10.1109/ICASSP.2011.5946307 


\title{
CHANNEL QUANTIZATION DESIGN IN MULTIUSER MIMO SYSTEMS: ASYMPTOTIC VERSUS PRACTICAL CONCLUSIONS
}

\author{
Emil Björnson, Konstantinos Ntontin, Björn Ottersten \\ ACCESS Linnaeus Center, KTH Royal Institute of Technology, SE-100 44 Stockholm, Sweden
}

\begin{abstract}
Feedback of channel state information (CSI) is necessary to achieve high throughput and low outage probability in multiuser multiantenna systems. There are two types of CSI: directional and quality information. Many papers have analyzed the importance of these in asymptotic regimes. However, we show that such results should be handled with care, as very different conclusions can be drawn depending on the spatial correlation and number of users. Therefore, we propose a quantization framework and evaluate the tradeoff between directional and quality feedback under practical conditions.
\end{abstract}

Index Terms - Finite rate feedback, multiplexing gain, multiuser MIMO, directional and quality quantization, $\epsilon$-outage rate

\section{INTRODUCTION}

Multiantenna techniques introduce a spatial dimension to wireless systems, enabling spatial multiplexing of multiple data streams with coordinated interference. In downlink cellular systems, these streams are assigned to different users, known as multiuser MIMO [1]. Compared with single-user systems, the multiuser performance is enhanced through multiuser diversity and by turning spatial correlation into an advantage [2]. However, multiuser MIMO requires channel state information (CSI) at the transmitter [1]. In frequencydivision duplex (FDD) systems, CSI is estimated at the receiver and then quantized and fed back to the transmitter.

CSI can be divided into two categories: channel directional information (CDI) and channel quality information (CQI). The directional part is useful for beamforming design, while the quality part is used for selecting data rates supported by the fading channel. Motivated by the future need for having many small cells with high signal-to-noise ratios (SNRs), asymptotic analysis has been used to evaluate the relative importance of CDI and CQI feedback. In [3], it is shown that accurate CDI is very important to achieve the optimal sum rate scaling, while CQI feedback can be replaced by long-term coding. Contrary, [4] shows that random beamforming with CQI feedback is sufficient to achieve the optimal sum rate scaling if the number of users is large. In fact, no CDI feedback is required in spatially correlated channels (with known channel statistics) if the number of users is large [5]. In other words, different conclusions can be drawn from asymptotics depending on system conditions.

The design of quantization codebooks for limited feedback systems have received much attention [1]. Optimal codebooks (for a given distortion measure) are achieved by the generalized Lloyd algorithm, but then a large set of codebooks are required to cover any possible situation. In [6], the correlated Grassmannian codebook

B. Ottersten is also with securityandtrust.lu, University of Luxembourg.

The research leading to these results has received funding from the European Research Council under the European Community's Seventh Framework Programme (FP7/2007-2013) / ERC Grant Agreement no. 228044. was proposed for CDI quantization. It is simple to adapt to the channel statistics under a minor performance loss. CQI feedback can be based on the actual coding rates supported by the system [7], but only parts of this list are relevant for each user if their long-term statistics are known. Thus, the transmission design and scheduling can be improved by feeding back a statistics-aware quality indicator such as the channel norm $[8]$ or the (approximated) expected rate $[9,10]$. It is common to assume perfect CQI feedback $[4,5,9]$ or long-term rate averaging without CQI feedback [3,6], but a framework for entropymaximizing CQI quantization was proposed in [8].

In this paper, we investigate the tradeoff between CDI and CQI feedback in multiuser MIMO systems. The contributions are:

- We analyze the asymptotic behavior of the $\epsilon$-outage sum rate and show that the full multiplexing gain can be achieved using only CDI feedback, only CQI feedback, or neither-all depending on the number of users and the spatial correlation.

- We propose a quantization framework with a novel combination of correlated Grassmannian codebooks for CDI feedback and entropy-maximizing quantization of the approximated rate. The impact of spatial correlation and of division of feedback bits on performance is investigated numerically.

\section{SYSTEM MODEL}

We consider a multiantenna downlink system. A base station with $N_{t}$ antennas communicates with $K$ single-antenna users $\left(K \geq N_{t}\right)$. The single-carrier channels are block and flat fading, for ease of exposition. The channel $\mathbf{h}_{k} \in \mathbb{C}^{N_{t} \times 1}$ to user $k$ is modeled as circular symmetric complex Gaussian and the received signal is modeled as

$$
y_{k}=\mathbf{h}_{k}^{H} \mathbf{x}+n_{k}, \quad \mathbf{h}_{k} \in \mathcal{C N}\left(\mathbf{0}, \mathbf{R}_{k}\right),
$$

where $\mathbf{x} \in \mathbb{C}^{N_{t} \times 1}$ is the transmitted signal and $n_{k} \in \mathcal{C N}(0,1)$ is white noise with normalized variance, for simplicity. The eigenvalue distribution of the channel covariance matrix $\mathbf{R}_{k} \in \mathbb{C}^{N_{t} \times N_{t}}$ describes the spatial correlation of the propagation environment. High correlation (i.e., a few dominating eigenvalues) often appear in widearea outdoor scenarios, while low correlation (small eigenvalue differences) can appear for indoor channels.

In each block, a subset $\mathcal{S} \subset\{1, \ldots, K\}$ of users are scheduled for transmission. Linear precoding is assumed [3,9,10], which is justified by the need for practical non-complex transceivers and its optimal asymptotic performance scaling [3]. Under these conditions, the signal-to-interference-and-noise ratio (SINR) for user $k \in \mathcal{S}$ is

$$
\operatorname{SINR}_{k}=\frac{P p_{k}\left|\mathbf{h}_{k}^{H} \mathbf{w}_{k}\right|^{2}}{1+\sum_{l \in \mathcal{S} \backslash\{k\}} P p_{l}\left|\mathbf{h}_{k}^{H} \mathbf{w}_{l}\right|^{2}}
$$

where $\mathbf{w}_{l} \in \mathbb{C}^{N_{t} \times 1}$ is the unit-norm beamforming vector towards user $l$. The total transmission power $P$ is divided among users by the splitting parameters $p_{1}, \ldots, p_{K} \geq 0$, which satisfy $\sum_{k=1}^{K} p_{k} \leq 1$. 


\section{1. $\epsilon$-Outage sum rate}

To model an FDD system, we assume a block structure where the channel vectors are: 1) Estimated at the receiver through training signaling; 2) Quantized and fed back to the transmitter; 3) Used for transmission until outdated. To concentrate on quantization and feedback design, user $k$ estimates the instantaneous channel $\mathbf{h}_{k}$ without error and the feedback channel is error-free. The transmitter knows the slowly-varying covariance matrix $\mathbf{R}_{k}$ and receives a finite number of bits describing the instantaneous channels $\mathbf{h}_{k}$ for all $k$.

An inherent problem in downlink communication is that users only know their own channels, while the transmitter have quantized CSI. Thus, the supported data rates in a block need to be estimated under CSI uncertainty, leading to a probability of outage ${ }^{1}$ (i.e., overestimation). Using hybrid ARQ, data sent in outage is not entirely lost, but the outage probability should be low to achieve efficiency.

Let $F_{\text {SINR }_{k}}(\cdot)$ be the cdf of $\operatorname{SINR}_{k}$, based on transmit-side CSI. Then, having an acceptable outage probability of $\epsilon>0$ means that

$$
\operatorname{Pr}\left\{\log _{2}\left(1+\operatorname{SINR}_{k}\right)<R_{k, \text { out }}\right\}=F_{\text {SINR }_{k}}\left(2^{R_{k, \text { out }}}-1\right)=\epsilon .
$$

To satisfy this probability, we thus transmit with the $\epsilon$-outage rate $R_{k, \text { out }}=\log _{2}\left(1+F_{\mathrm{SINR}_{k}}^{-1}(\epsilon)\right)$ to user $k$. The $\epsilon$-outage sum rate is

$$
R_{\text {sum }}(P, \epsilon)=\sum_{k \in \mathcal{S}} R_{k, \text { out }}=\sum_{k \in \mathcal{S}} \log _{2}\left(1+F_{\text {SINR }_{k}}^{-1}(\epsilon)\right) .
$$

In Section 3, the $\epsilon$-outage sum rate is analyzed asymptotically, while a practical quantization/precoding scheme is evaluated in Section 4.

\section{ASYMPTOTIC SUM RATE: MULTIPLEXING GAIN}

In the desirable high SNR regime, the performance is governed by the scaling of the $\epsilon$-outage sum rate with SNR. This scaling is characterized by the multiplexing gain (or pre-log factor) [3], defined as

$$
\lim _{P \rightarrow \infty} \frac{R_{\text {sum }}(P, \epsilon)}{\log _{2}(P)}=r .
$$

Thus, having a multiplexing gain of $r$ means that the $\epsilon$-outage sum rate behaves as $r \log _{2}(P)+$ constant at high transmit power. The multiplexing gain in a multiuser system is limited by the spatial degrees of freedom in the systems, thus $r \leq \min \left(N_{t}, K\right)$. As we have assumed $N_{t} \leq K$, we say that $N_{t}$ is the full multiplexing gain.

To achieve the full multiplexing gain, it is necessary that the interference term $\sum_{l \in \mathcal{S} \backslash\{k\}} P p_{l}\left|\mathbf{h}_{k}^{H} \mathbf{w}_{l}\right|^{2}$ in each $\operatorname{SINR}_{k}$ is bounded by a constant as $P \rightarrow \infty$. It was proved in [3] that this is achieved by zero-forcing precoding if accurate CDI is available. The author of [3] avoids CQI feedback by considering the long-term average rate, but a similar result can be achieved for the $\epsilon$-outage sum rate:

Theorem 1. For any selection of $N_{t}$ users, let the channel directions $\overline{\mathbf{h}}_{k}=\frac{\mathbf{h}_{k}}{\left\|\mathbf{h}_{k}\right\|}$ be known to the transmitter while the gains $\left\|\mathbf{h}_{k}\right\|^{2}$ are unknown. With probability one, zero-forcing precoding makes the $\epsilon$-outage sum rate achieve the full multiplexing gain of $N_{t}$.

Proof. With probability one, the channel directions are different: $\left|\overline{\mathbf{h}}_{l}^{H} \overline{\mathbf{h}}_{k}\right|<1 \forall l \neq k$. Then, zero-forcing precoding exists and can be used to cancel out all co-user interference. Let $\mathbf{w}_{k}^{(\mathrm{ZF})}$ denote the zero-forcing beamformer of user $k$. For an arbitrary $p_{k}>0$,

$$
\begin{aligned}
F_{\mathrm{SINR}_{k} \mid \overline{\mathbf{h}}_{k}}(x) & =\operatorname{Pr}\left\{P p_{k}\left\|\mathbf{h}_{k}\right\|^{2}\left|\overline{\mathbf{h}}_{k}^{H} \mathbf{w}_{k}^{(\mathrm{ZF})}\right|^{2} \leq x\right\} \\
& =F_{\left\|\mathbf{h}_{k}\right\|^{2} \mid \overline{\mathbf{h}}_{k}}\left(\frac{x}{P p_{k}\left|\overline{\mathbf{h}}_{k}^{H} \mathbf{w}_{k}^{(\mathrm{ZF})}\right|^{2}}\right)=\epsilon .
\end{aligned}
$$

\footnotetext{
${ }^{1}$ Outage can be avoided by considering the long-term average throughput, but this is infeasible under delay-constraints and rapid user selection.
}

The second equality follows from using the (conditional) cdf of $\left\|\mathbf{h}_{k}\right\|^{2}$, which is the only random variable when $\overline{\mathbf{h}}_{k}$ is known. This cdf can be derived using Bayes' theorem and by rewriting the pdf of a complex Gaussian vector in a similar manner as in [5]:

$$
F_{\left\|\mathbf{h}_{k}\right\|^{2} \mid \overline{\mathbf{h}}_{k}}(x)=1-e^{-x \overline{\mathbf{h}}_{k}^{H} \mathbf{R}_{k}^{-1} \overline{\mathbf{h}}_{k}} \sum_{m=0}^{N_{t}-1} \frac{\left(x \overline{\mathbf{h}}_{k}^{H} \mathbf{R}_{k}^{-1} \overline{\mathbf{h}}_{k}\right)^{m}}{m !} .
$$

Using this expression, we can evaluate the $\epsilon$-outage rate expression (defined in (3) and (4)) by inverting the cdf expression from (6):

$$
\begin{aligned}
R_{k, \text { out }} & =\log _{2}\left(1+F_{\mathrm{SINR}_{k} \mid \overline{\mathbf{h}}_{k}}^{-1}(\epsilon)\right) \\
& =\log _{2}\left(1+P p_{k}\left|\overline{\mathbf{h}}_{k}^{H} \mathbf{w}_{k}^{(\mathrm{ZF})}\right|^{2} F_{\left\|\mathbf{h}_{k}\right\|^{2} \mid \overline{\mathbf{h}}_{k}}^{-1}(\epsilon)\right) .
\end{aligned}
$$

For all $\epsilon>0, F_{\left\|\mathbf{h}_{k}\right\|^{2} \mid \overline{\mathbf{h}}_{k}}^{-1}(\epsilon)$ is a strictly positive constant. Thus, $R_{k, \text { out }}$ behaves as $\log _{2}(P)+$ constant at high $P$. By repeating this for all the $N_{t}$ users, we conclude that the $\epsilon$-outage sum rate achieves the full multiplexing gain of $N_{t}$.

Based on this theorem (and [3]), one might believe that CDI feedback is of dominating importance in multiuser MIMO systems. But apart from the multiplexing gain, the high SNR performance is also characterized by the (constant) high-SNR offset [11]. This parameter depends on CQI feedback and might have great impact at practical SNRs. In addition, Theorem 1 is not saying whether the full multiplexing gain is achievable under other conditions, for instance, using only CQI feedback. Remarkably, it is achieved if a set of fixed beamforming vectors are used and the number of users is large [4], such that each beamformer closely models the channel of some user. The long-term average rate was considered in [4], based on feedback of the index of the best beamforming vector and the corresponding channel quality. The next theorem shows a similar result for the $\epsilon$ outage sum rate using only CQI feedback and beamforming based on the dominating eigenvector $\mathbf{u}_{k}$ (or one of them) of each $\mathbf{R}_{k}$.

Theorem 2. Assume that the transmitter knows the quality indicator

$$
\gamma_{k}=\frac{P\left|\mathbf{h}_{k}^{H} \mathbf{u}_{k}\right|^{2}}{1+P\left(\left\|\mathbf{h}_{k}\right\|^{2}-\left|\mathbf{h}_{k}^{H} \mathbf{u}_{k}\right|^{2}\right)}
$$

for all $k=1, \ldots, K$, while the channel directions $\frac{\mathbf{h}_{k}}{\left\|\mathbf{h}_{k}\right\|}$ are unknown. If all $\mathbf{R}_{k}$ have the same dominating eigenvalue $\lambda_{1}>0$ but different eigenvectors ${ }^{2}$, then beamforming exploiting only statistics can (with probability one) make the $\epsilon$-outage sum rate achieve the full multiplexing gain of $N_{t}$ if $K \rightarrow \infty$ such that $\frac{P}{\log (K)} \rightarrow c<\infty$.

Proof. For any user selection with $|\mathcal{S}|=N_{t}$, the dominating eigenvectors $\left\{\mathbf{u}_{k}\right\}_{k \in \mathcal{S}}$ satisfy $\left|\mathbf{u}_{k}^{H} \mathbf{u}_{l}\right|<1 \forall l \neq k$. Thus, there exists (zero-forcing) beamforming vectors $\left\{\widetilde{\mathbf{w}}_{k}\right\}_{k \in \mathcal{S}}$ such that $\mathbf{u}_{k}^{H} \widetilde{\mathbf{w}}_{l}=0$ for $l \neq k$. Assuming $p_{k}>0$, the SINR of user $k$ can be bounded as

$$
\begin{aligned}
\frac{P p_{k}\left|\mathbf{h}_{k}^{H} \widetilde{\mathbf{w}}_{k}\right|^{2}}{1+\sum_{l \in \mathcal{S} \backslash\{k\}} P p_{l}\left|\mathbf{h}_{k}^{H} \widetilde{\mathbf{w}}_{l}\right|^{2}} & \geq \frac{P p_{k} \beta_{k}\left|\mathbf{h}_{k}^{H} \mathbf{u}_{k}\right|^{2}}{1+P\left(\left\|\mathbf{h}_{k}\right\|^{2}-\left|\mathbf{h}_{k}^{H} \mathbf{u}_{k}\right|^{2}\right)} \\
& =p_{k} \beta_{k} \gamma_{k}>0
\end{aligned}
$$

for some $\beta_{k}>0$ satisfying $\left|\mathbf{h}_{k}^{H} \widetilde{\mathbf{w}}_{k}\right|^{2} \geq \beta_{k}\left|\mathbf{h}_{k}^{H} \mathbf{u}_{k}\right|^{2}$ (with probability one), by pretending that all co-user transmissions create maximal interference, and by identifying the quality indicator $\gamma_{k}$ that is known to the transmitter. If we can show that $\gamma_{k}$ behaves as $P$ at high $P$ (and increasing $K$ ), we have proved the multiplexing gain.

\footnotetext{
${ }^{2}$ Satisfied for the exponential model with equal antenna correlation and random user directions [8], but is merely assumed to simplify the derivation.
} 


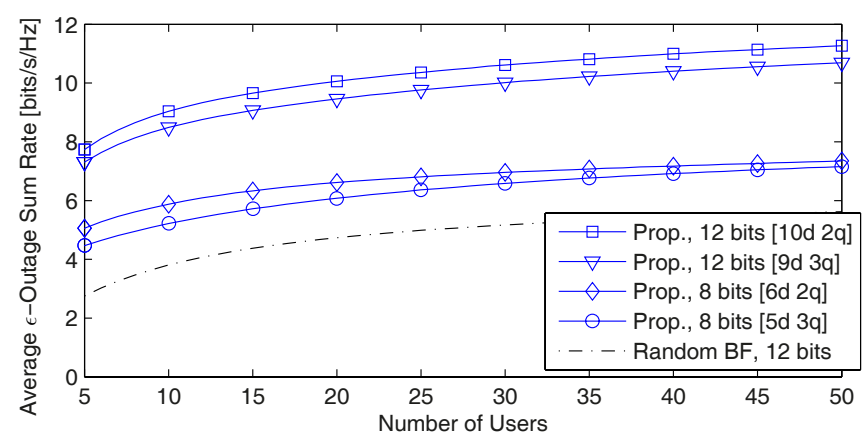

Fig. 1. Average $\epsilon$-outage sum rate as a function of number of users.

For notational convenience, assume there are $N_{t} K$ users, which are divided into $N_{t}$ equally large groups. In each group, we schedule the user with the largest $\gamma_{k}$. Applying the extreme value theory of Theorem A.2 in [4], we conclude that

$$
\begin{aligned}
\operatorname{Pr}\left\{\max _{1 \leq k \leq K} \gamma_{k} \geq P \lambda_{1} \log (K)\right. & -P N_{t} \lambda_{1} \log (P \log (K)) \\
& +\mathcal{O}(1)\} \geq 1-\mathcal{O}\left(1 / K^{P}\right) .
\end{aligned}
$$

We omit the derivation for space limitations, but it follows along the lines of Corollary A.1 in [4] with the exception that $P$ is not a constant and by observing that $\operatorname{Pr}\left\{\max _{1<k<K} \gamma_{k} \geq c\right\}$ is lower bounded (for any $c$ ) by the case when $\mathbf{R}_{k}=\lambda_{1} \mathbf{I}$ for all $k$.

If $\frac{P}{\log (K)} \rightarrow c<\infty$, then $P \log (K)-P N_{t} \log (P \log (K))$ is dominated by $P \log (K)$. For sufficiently large $K$ and $P$, the rate of the user with the largest $\gamma_{k}$ scales as $\log _{2}(P \log (K))$ with a probability larger than $1-\epsilon$. This holds for the user with the largest $\gamma_{k}$ in each of the $N_{t}$ groups, yielding a total multiplexing gain of $N_{t}$.

Some scepticism is in order when interpreting Theorem 2; the number of users should scale exponentially fast (compared with $P$ ) to find users with $\frac{\mathbf{h}_{k}}{\left\|\mathbf{h}_{k}\right\|} \approx \mathbf{u}_{k}$. Then, a multiuser diversity gain also appears since the strongest channel gain approaches infinity [4], which basically is a modeling artifact of Rayleigh fading channels [12]. Still, the performance under pure CQI feedback might be good at practical SNRs and number of users. When feedback quantization is considered, one should keep in mind that the one-dimensional CQI is easier to quantize accurately than the $N_{t}$-dimensional CDI. Another important factor is spatial correlation; the channel direction is more or less given by the long-term statistics in strongly correlated environments. Theorem 3 shows that the full multiplexing gain can be achieved without any feedback if the correlation is strong.

Theorem 3. Assume that the transmitter only has statistical CSI and that all $\mathbf{R}_{k}$ have different dominating eigenvectors $\mathbf{u}_{k}$. Denote the eigenvalues as $\lambda_{k, 1} \geq \ldots \geq \lambda_{k, N_{t}}$. With probability one, beamforming only exploiting statistics can achieve the full multiplexing gain of $N_{t}$ if $\frac{\lambda_{k, 1}}{\lambda_{k, 2}} \rightarrow \infty$ such that $\frac{P}{\lambda_{k, 1} / \lambda_{k, 2}} \rightarrow c_{k}<\infty$ for all $k$.

Proof. Select $N_{t}$ users with different dominating eigenvectors $\mathbf{u}_{k}$ for all $k \in \mathcal{S}$. Then, there exists (zero-forcing) beamforming vectors $\left\{\widetilde{\mathbf{w}}_{k}\right\}_{k \in \mathcal{S}}$ such that $\mathbf{u}_{k}^{H} \widetilde{\mathbf{w}}_{l}=0$ for $l \neq k$. With probability one, $\left|\mathbf{h}_{k}^{H} \widetilde{\mathbf{w}}_{k}\right|>0$. Thus, the signal term in $\operatorname{SINR}_{k}$ scales like $P \lambda_{k, 1}$ while the interference term scales as $P \lambda_{k, 2}$. If $\frac{P}{\lambda_{k, 1} / \lambda_{k, 2}} \rightarrow c_{k}<$ $\infty$, then the signal term goes to infinity with $P$ while the interference term is bounded. Thus, the full multiplexing gain is achieved.

The bottom line is that asymptotic analysis can provide very diverse answers to feedback design questions in multiuser MIMO systems. The actual performance depends on the relationship between SNR, number of users, spatial correlation, and feedback design.

\section{PRACTICAL FEEDBACK QUANTIZATION}

Next, we propose and evaluate a practical quantization framework based on correlated Grassmannian codebooks for CDI feedback and entropy-maximizing CQI feedback. The CDI and CQI are quantized separately using $d$ and $q$ bits, respectively, with $b=d+q$. This separation is suboptimal but necessary to achieve a simple framework and to investigate the tradeoff between CDI and CQI feedback.

Based on the system model, the receivers cannot predict the couser interference at the point of feedback. Therefore, the quantization will be based on an (approximative) lower bound on the SINR:

$$
\operatorname{SINR}_{k} \gtrsim \frac{\frac{P}{N_{t}}\left|\mathbf{h}_{k}^{H} \mathbf{w}_{k}\right|^{2}}{1+\frac{P}{N_{t}}\left(\left\|\mathbf{h}_{k}\right\|^{2}-\left|\mathbf{h}_{k}^{H} \mathbf{w}_{k}\right|^{2}\right)}=\nu_{k}\left(\mathbf{w}_{k}\right) .
$$

It is achieved by assuming equal power allocation, $|\mathcal{S}|=N_{t}$, and that interference is equally spread in the space orthogonal to $\mathbf{w}_{k}$ $[9,10]$. The proposed feedback scheme is based on $\nu_{k}\left(\mathbf{w}_{k}\right)$.

\subsection{CDI quantization: Correlated Grassmannian codebook}

Similar to [3, 6, 9], the channel direction $\overline{\mathbf{h}}_{k}=\frac{\mathbf{h}_{k}}{\left\|\mathbf{h}_{k}\right\|}$ is quantized using a codebook $\mathcal{D}_{k}=\left\{\mathbf{v}_{k, 1}, \ldots, \mathbf{v}_{2^{k, d}}\right\}$ of $2^{d}$ of vectors on the $N_{t}$-dimensional complex unit sphere. In each block, user $k$ selects the vector in $\mathcal{D}_{k}$ that maximizes $\nu_{k}(\cdot)$ and feeds back the index

$$
j_{k}=\underset{i=1, \ldots, 2^{\left|\mathcal{D}_{k}\right|}}{\arg \max } \nu_{k}\left(\mathbf{v}_{k, i}\right)=\underset{i=1, \ldots, 2^{\left|\mathcal{D}_{k}\right|}}{\arg \min }\left\|\mathbf{h}_{k}\right\|^{2}-\left|\mathbf{h}_{k}^{H} \mathbf{v}_{k, i}\right|^{2} .
$$

The equality holds since the numerator and denominator achieve their optimal values simultaneously. Based on [3], we use the Grassmannian codebook $\mathcal{G}=\left\{\mathbf{g}_{1}, \ldots, \mathbf{g}_{2^{d}}\right\}$ that maximizes the minimal angle between the codeword vectors $\mathbf{g}_{l}$ and rotate it according to each user's long-term statistics: $\mathbf{v}_{k, l}=\mathbf{R}_{k}^{1 / 2} \mathbf{g}_{l} /\left\|\mathbf{R}_{k}^{1 / 2} \mathbf{g}_{l}\right\| \forall l$.

\subsection{CQI quantization: Feedback-entropy maximization}

To maximize the CQI feedback information, the $2^{q}$ quantization intervals should be equally probable. The entropy-maximizing framework in $[8$, Lemma 1] divides the space $[0, \infty)$ of CQI values into disjoint intervals based on the cdf $F_{k}(x)$ of the feedback parameter. The $i$ th interval is $\left[a_{k, i-1}, a_{k, i}\right)$ with $a_{k, i}=0, a_{k, 2^{q}}=\infty$, and

$$
a_{k, i}=F_{k}^{-1}\left(\frac{i}{2^{q}}\right) \quad i=1, \ldots, 2^{q}-1 .
$$

By construction, these intervals are equally probable. It remains to characterize the cdf of $\nu_{k}\left(\mathbf{v}_{k, j_{k}}\right)$, which unfortunately is hard to do explicitly since the index $j_{k}$ originates from (13). In the evaluation, we use a set of channel realizations to generate $F_{k}(\cdot)$ numerically.

\subsection{Scheduling, precoding, and rate adaptation}

We adopt the user selection scheme of [10, Algorithm 1] for joint scheduling and precoding. This scheme combines zero-forcing precoding $\left\{\mathbf{w}_{k}^{\mathrm{ZF}}(\mathcal{S})\right\}_{k \in \mathcal{S}}$ with greedy selection of the user set $\mathcal{S}$. Based on feedback, the SINR of user $k \in \mathcal{S}$ is estimated as $\widehat{\operatorname{SINR}}_{k}=N_{t}\left|\mathbf{v}_{k, j_{k}}^{H} \mathbf{w}_{k}^{\mathrm{ZF}}(\mathcal{S})\right|^{2} \nu_{k}\left(\mathbf{v}_{k, j_{k}}\right) /|\mathcal{S}|$. Rate adaptation is required to mitigate CSI uncertainty and achieve a target outage probability. To this end, we use a fade-margin $\alpha_{k}$ that is selected numerically to make $\operatorname{Pr}\left\{\log _{2}\left(1+\operatorname{SINR}_{k}\right)<\log _{2}\left(1+\alpha_{k} \widehat{\operatorname{SINR}}_{k}\right)\right\}=\epsilon$. 


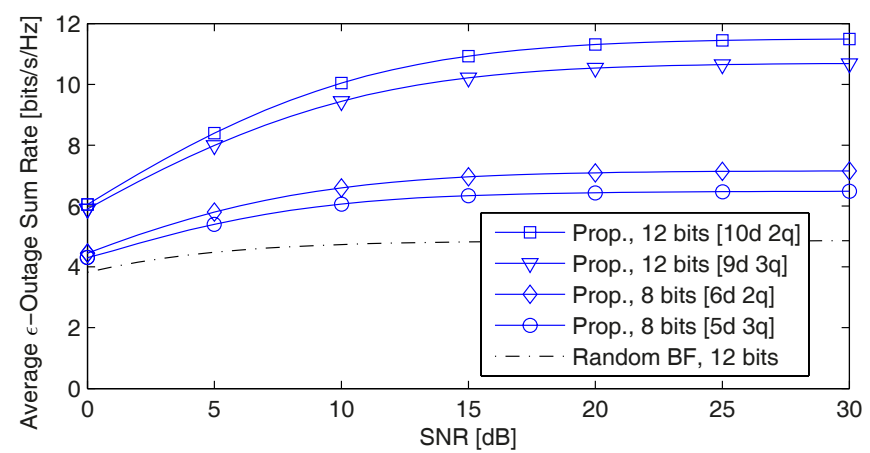

Fig. 2. Average $\epsilon$-outage sum rate as a function of the SNR.

\subsection{Numerical evaluation}

Next, we will evaluate the performance of this simple quantization framework and compare it with random beamforming [4] and normsupported eigenbeamforming $[5,8]$. To concentrate on the tradeoff between CDI and CQI feedback, we consider a simple setup where the users are equally spaced on a circle around a base station with $N_{t}=4$ antennas. Hence, users have identical average SNRs, defined as $\frac{P}{N_{t}} \operatorname{tr}\left(\mathbf{R}_{k}\right)$. The outage probability is $5 \%$ (i.e., $\epsilon=0.05$ ).

In Fig. 1, we consider an uncorrelated scenario (i.e., $\mathbf{R}_{k} \propto \mathbf{I}$ ) with an SNR of $10 \mathrm{~dB}$ and either $b=8$ or $b=12$ feedback bits per user. The performance increases with the number of users, but the highest $\epsilon$-outage sum rate is always achieved by allocating 2 bits for CQI feedback and the remaining bits for CDI feedback. The proposed scheme can provide much better performance than random beamforming. In fact, random beamforming performs rather poorly and the optimal asymptotic behavior proved in Theorem 2 has little impact on the performance at practical number of users.

The same setup is considered in Fig. 2, but the number of users is fixed at $K=20$ and the SNR is varied. The performance improves with the SNR and it becomes increasingly important with CDI feedback. The system is interference-limited at high SNR, meaning that the sum rate does not approach infinity. This confirms [3], where it was shown that the number of feedback bits should increase with SNR to achieve the full multiplexing gain. If $b$ is fixed, the maximal multiplexing gain is one, achieved by single user transmission [10].

An outdoor scenario is considered in Fig. 3 using the local scattering model with a uniform circular array and half a wavelength antenna separation $[5,8]$. The angular spread (as seen from the base station) describes the spatial correlation-small spread means high correlation. The SNR is $10 \mathrm{~dB}$ and $b=8$ feedback bits are used per user. In highly correlated scenarios, we observe a higher importance of CQI feedback than in the uncorrelated case. This is mainly since the covariance matrix $\mathbf{R}_{k}$ already provides much CDI [5]. The norm-supported eigenbeamforming from [5,8] demonstrates that good performance is achievable in highly correlated systems using only CQI feedback. As the angular spread increases, CDI feedback is necessary to pertain high performance, but the difference between the three feedback divisions is minor. Random beamforming ignores the correlation and is therefore almost unaffected by it.

\section{CONCLUSION}

We have proved that depending on the number of users and spatial correlation, the full multiplexing gain can be achieved with only CDI feedback, only CQI feedback, or without any feedback. Thus, asymptotic analysis provides very diverse answers to the tradeoff between CDI and CQI feedback in practical systems. We have an-

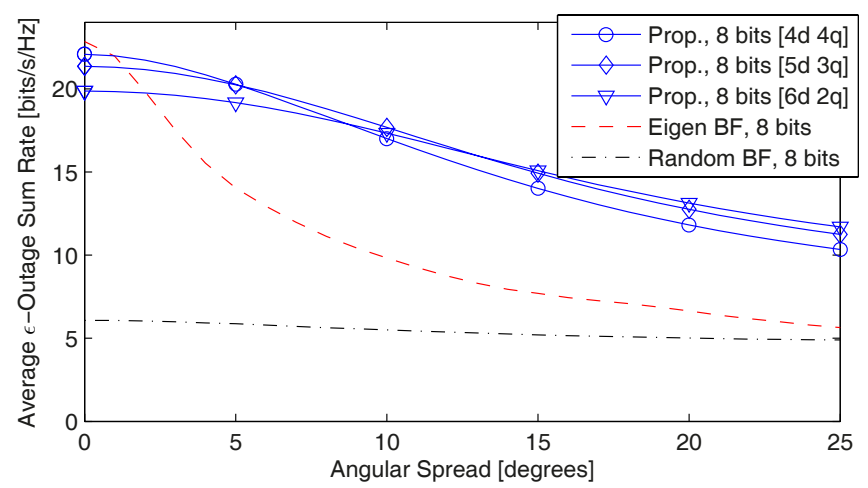

Fig. 3. Average $\epsilon$-outage sum rate as a function of the angular spread.

alyzed this tradeoff numerically and seen that 2-3 bits of CQI feedback typically is sufficient, while the remaining bits should be used for CDI feedback. The importance of CQI increases with the spatial correlation, while the need for CDI increases with SNR.

\section{REFERENCES}

[1] D.J. Love, R.W. Heath, V.K.N. Lau, D. Gesbert, B.D. Rao, and M. Andrews, "An overview of limited feedback in wireless communication systems," IEEE J. Sel. Areas Commun., vol. 26, no. 8, pp. 1341-1365, 2008.

[2] H. Wang, P. Wang, L. Ping, and X. Lin, "On the impact of antenna correlation in multi-user MIMO systems with rate constraints," IEEE Commun. Lett., vol. 13, pp. 935-937, 2009.

[3] N. Jindal, "MIMO broadcast channels with finite-rate feedback," IEEE Trans. Inf. Theory, vol. 52, pp. 5045-5060, 2006.

[4] M. Sharif and B. Hassibi, "On the capacity of MIMO broadcast channels with partial side information," IEEE Trans. Inf. Theory, vol. 51, no. 2, pp. 506-522, 2005.

[5] D. Hammarwall, M. Bengtsson, and B. Ottersten, "Acquiring partial CSI for spatially selective transmission by instantaneous channel norm feedback," IEEE Trans. Signal Process., vol. 56, no. 3, pp. 1188-1204, 2008.

[6] D.J. Love and R.W. Heath, "Limited feedback diversity techniques for correlated channels," IEEE Trans. Veh. Technol., vol. 55, no. 2, pp. 718-722, 2006.

[7] Evolved Universal Terrestrial Radio Access (E-UTRA); Physical Channels and Modulation (Release 9), 3GPP TS 36.213.

[8] E. Björnson, D. Hammarwall, and B. Ottersten, "Exploiting quantized channel norm feedback through conditional statistics in arbitrarily correlated MIMO systems," IEEE Trans. Signal Process., vol. 57, no. 10, pp. 4027-4041, 2009.

[9] T. Yoo, N. Jindal, and A. Goldsmith, "Multi-antenna downlink channels with limited feedback and user selection," IEEE J. Sel. Areas Commun., vol. 25, no. 7, pp. 1478-1491, 2007.

[10] M. Trivellato, F. Boccardi, and F. Tosato, "User selection schemes for MIMO broadcast channels with limited feedback," in Proc. IEEE VTC'07-Spring, 2007, pp. 2089-2093.

[11] A. Lozano, A.M. Tulino, and S. Verdú, "High-SNR power offset in multiantenna communication," IEEE Trans. Inf. Theory, vol. 51, no. 12, pp. 4134-4151, 2005.

[12] M. Dohler, R.W. Heath, A. Lozano, C. Papadias, and R.A. Valenzuela, "Is the PHY layer dead?," IEEE Commun. Mag., to appear. 